\title{
Magnetically induced texture development in zinc alloy sheet
}

\author{
A.D. Sheikh-Ali ${ }^{\text {a, } * 1}$, D.A. Molodov ${ }^{\text {b }}$, H. Garmestani ${ }^{\text {a }}$ \\ a Laboratory for Micromechanics of Materials, FSU-National High Magnetic Field Laboratory, 1800 East Paul Dirac Drive, \\ Tallahassee, Florida 32310, USA \\ ${ }^{\mathrm{b}}$ Institut für Metallkunde und Metallphysik, RWTH Aachen, D-52056 Aachen, Germany
}

Received 15 January 2002; received in revised form 5 February 2002; accepted 4 March 2002

\begin{abstract}
Highly textured $\mathrm{Zn}-1.1 \% \mathrm{Al}$ sheet was annealed in a direct-current magnetic field of $25.5 \mathrm{MA} / \mathrm{m}$. Depending on the orientation to the field the texture components strengthened, retained their original intensity or disappeared. The results obtained are interpreted in terms of magnetically induced selective grain growth. @ 2002 Published by Elsevier Science Ltd. on behalf of Acta Materialia Inc.
\end{abstract}

Keywords: Texture; Magnetic susceptibility; Boundary migration; Grain growth; Microstructure

\section{Introduction}

It has been shown that magnetic fields can affect or initiate grain boundary motion (migration) in bismuth bicrystals and coarse-grained polycrystals $[1,2]$. In magnetically anisotropic materials the additional driving force for boundary migration (grain growth) is exerted by the difference in the magnetic free energy in differently oriented neighboring grains. In most of the anisotropic diamagnetic materials that difference is significantly smaller than in bismuth. Therefore, magnetically induced boundary migration in these materials can be expected in the case of sufficiently high magnetic fields. The objective of the current study is to

\footnotetext{
${ }^{*}$ Corresponding author.

E-mail address: sheikh@magnet.fsu.edu (A.D. Sheikh-Ali).

${ }^{1}$ On leave from Institute for Metals Superplasticity, Russian Academy of Sciences, 39 Khalturin Street, Ufa 450001, Russian Federation.
}

investigate texture changes in a $\mathrm{Zn}-1.1 \% \mathrm{Al}$ alloy during annealing in a strong magnetic field.

\section{Experimental procedure}

$\mathrm{Zn}-1.1 \% \mathrm{Al}$ alloy was prepared from high purity metals $(99.995 \% \mathrm{Zn}$ and $99.99 \% \mathrm{Al})$. The ingot was homogenized at $623 \mathrm{~K}$ for $150 \mathrm{~h}$, rolled at 573 $\mathrm{K}$ with the reduction of $50 \%$, and finally rolled at room temperature up to total reduction of $99 \%$. For each pass reduction in thickness was about $5 \%$. The direction of rolling was reversed after each pass. The final sheet thickness was $0.5 \mathrm{~mm}$. In order to prevent coarsening of Al-rich phase the material was stored in refrigerator at $203 \mathrm{~K}$ before the annealing. The experiments were carried out using a resistive, steady state $32 \mathrm{~T}$ Bitter magnet with a $32 \mathrm{~mm}$ bore diameter. The samples of $\mathrm{Zn}-$ $1.1 \% \mathrm{Al}$ alloy were annealed at a temperature of $663 \mathrm{~K}$ in a magnetic field of $32 \mathrm{~T}$. The annealing 


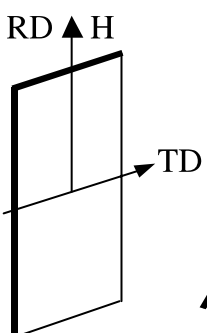

(a)

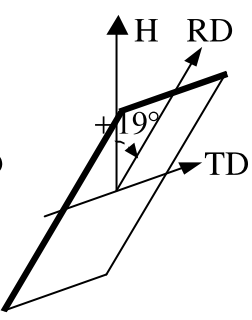

(b)

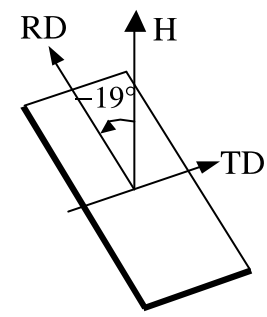

(c)
Fig. 1. Orientations of specimens with respect to the field during annealing. (a) Rolling direction is parallel to the field, (b) and (c) rolling direction is tilted from the direction of the field at $+19^{\circ}$ and $-19^{\circ}$, respectively.

time was $55 \mathrm{~min}$. Pole figures from the surface area of each sheet sample were determined by the Schulz method using $\mathrm{CuK}_{\alpha}$ radiation and Philips texture goniometer before and after annealing. For the sake of statistically reliable data the sample oscillation of $10 \mathrm{~mm}$ was used during texture measurements. Pole figures were calculated using Philips X'Pert Texture software through the calculation of orientation distribution functions on the basis of five different raw pole figures. The specimens were oriented differently with respect to the magnetic field (Fig. 1). For one specimen RD coincides with the direction of magnetic strength $H$ (Fig. 1(a)) and for the other set of specimens $\mathrm{RD}$ is tilted at $+19^{\circ}$ (Fig. 1(b)) and $-19^{\circ}$ (Fig. 1(c)) to the field direction.

\section{Results}

Fig. 2 illustrates $\left(\begin{array}{llll}0 & 0 & 0 & 2\end{array}\right)$ pole figure $\mathrm{Zn}-1.1 \% \mathrm{Al}$ sheet after $99 \%$ rolling. As seen from this pole

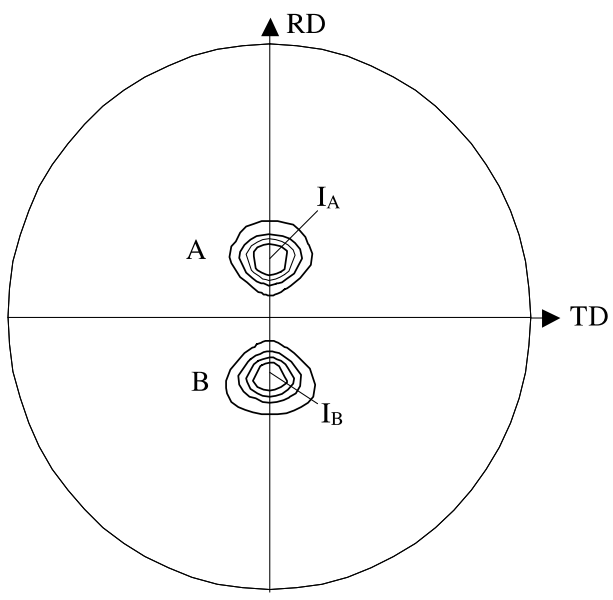

Fig. 2. Rolling texture ( 0002 pole figure) of $\mathrm{Zn}-1.1 \% \mathrm{Al}$ sheet.

figure two components with $c$-axis (basal poles) are tilted at some $15-20^{\circ}$ from normal direction to the rolling direction (RD) around the transverse direction (TD). The texture intensities for different specimens are varied from 28.0 to 34.0 for component A and from 20.2 to 24.9 for component B (Table 1). Annealing of specimens without field slightly changes intensities of the texture components and retains the original type of pole figure after rolling (Fig. 3(a)). Splitting of the texture components into a few subcomponents is observed after annealing with and without field. Annealing in magnetic field changes the type of pole figure. When RD is parallel to the field there is some unification of two peaks into one although the positions of the most intense subcomponents corresponds to the positions of the original components (Fig. 3(b)). During magnetic annealing of specimens tilted at $+19^{\circ}$ from the direction of the

Table 1

Intensities of basal poles before annealing and their orientations to the field direction during annealing

\begin{tabular}{llllll}
\hline $\begin{array}{l}\text { Specimen } \\
\text { no. }\end{array}$ & $I_{\mathrm{A}}$ & $I_{\mathrm{B}}$ & $\begin{array}{l}\text { Orientation of the specimen to } \\
\text { magnetic field during annealing }\end{array}$ & & \multicolumn{2}{l}{ Orientations of the $c$-axes to the field } \\
\cline { 5 - 6 } & & 20.2 & RD is parallel to $H$, (Fig. 1(a)) & $70-75^{\circ}$ & $70-75^{\circ}$ \\
7.2 & 32.9 & 24.9 & RD is at 19 to H, (Fig. 1(b)) & $89-94^{\circ}$ & $51-56^{\circ}$ \\
7.4 & 28.0 & 20.3 & RD is at $-19^{\circ}$ to $H$, (Fig. 1c)) & $51-56^{\circ}$ & $89-94^{\circ}$ \\
7.8 & 34.0 & 23.9 & Without field & - & - \\
\hline
\end{tabular}




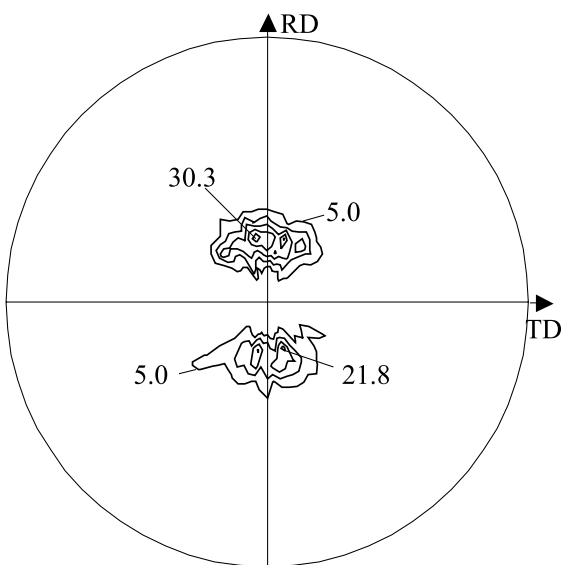

(a)

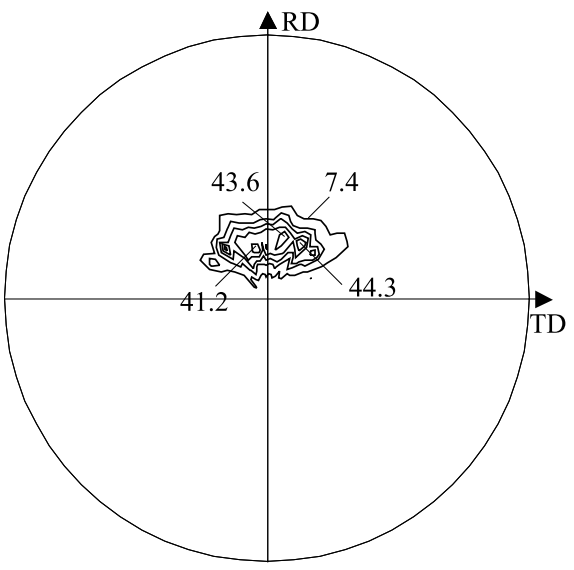

(c)

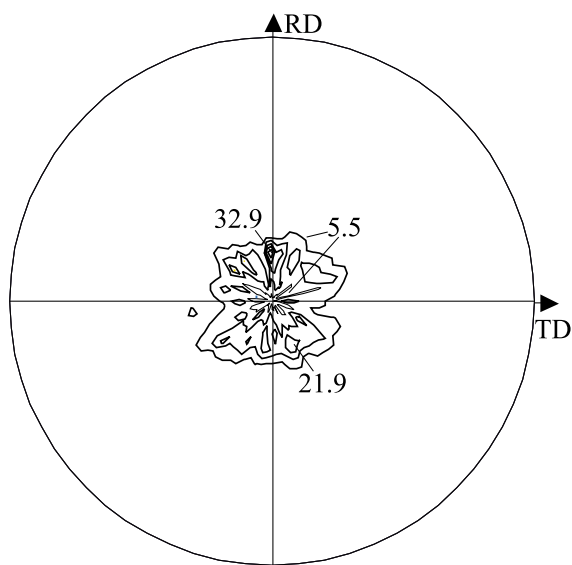

(b)

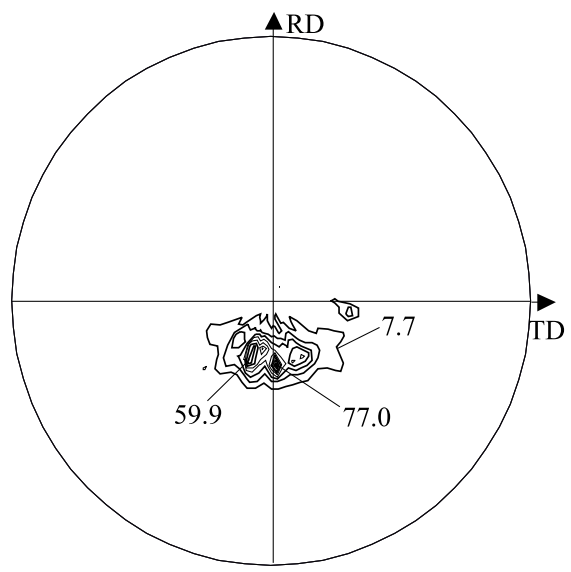

(d)

Fig. 3. Pole figures of $\mathrm{Zn}-1.1 \% \mathrm{Al}$ sheet specimens after annealing: (a) without field; (b) oriented parallel to the field; (c) tilted at $+19^{\circ}$ to the field about the TD and (d) tilted at $-19^{\circ}$ to the field about the TD.

field component B is totally annihilated while the intensity of component $\mathrm{A}$ rises in a factor of 1.5 comparing to the intensity of the original component A (Fig. 3(c)). In the case of magnetic annealing of specimen tilted at $-19^{\circ}$ from $H$ component A disappears completely and component $\mathrm{B}$ becomes 3.8 times as much as the intensity of the original component B (Fig. 3(d)). Fig. 4 illustrates specimen microstructures after rolling and after magnetic annealing. The grain size in the sheet surface obtained by linear intercept method is close to $2 \mu \mathrm{m}$. Annealing results in the increase of the average grain size up to $\sim 150 \mu \mathrm{m}$.

\section{Discussion}

The obtained results demonstrate that annealing of $\mathrm{Zn}-1.1 \% \mathrm{Al}$ sheet without field makes a minor change in texture. Retaining type of pole figure and intensity of texture peaks during annealing without field can be predicted for the most hexagonal materials having no phase transformations. In contrast, the annealing in a high magnetic field drastically changes the texture dependent on the orientation of the specimen with respect to the direction of the magnetic field. Table 1 shows orientations of specimens and texture components 

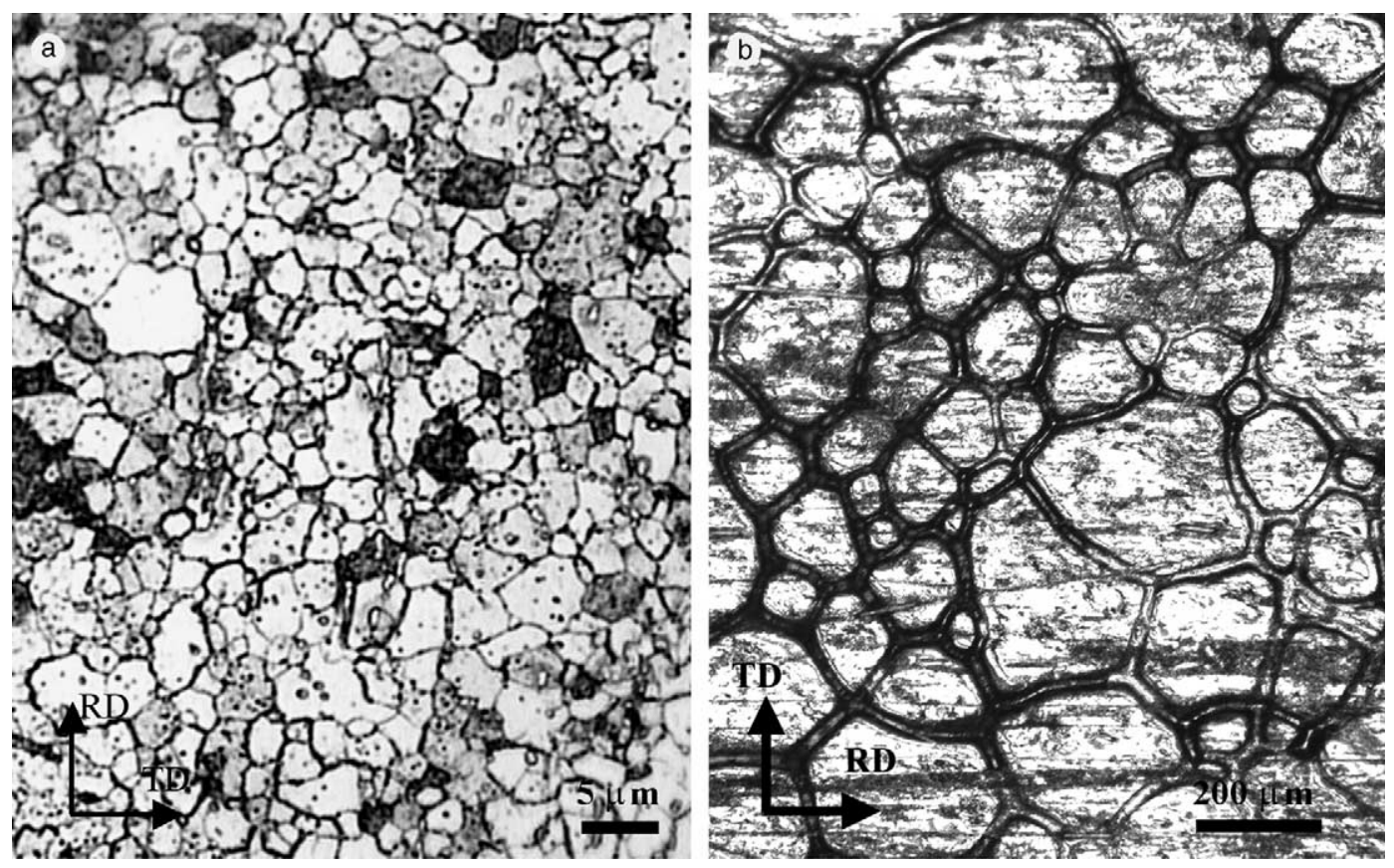

Fig. 4. Micrographs of $\mathrm{Zn}-1.1 \% \mathrm{Al}$ sheet after: (a) rolling and (b) magnetic annealing. Microstructure revealed by: (a) chemical etching and (b) thermal grooving of grain boundaries at the surface.

during magnetic annealing. For different specimens shown in Fig. 1 basal poles are tilted at three different angles to the field. For the specimen with RD parallel to the direction of the magnetic field $H$ (Fig. 1(a)), the angle between the $c$-axes of both components and the direction of the field is about $70-75^{\circ}$. The tilt of RD at $+19^{\circ}$ from the direction of the field (Fig. 2(b)) makes the $c$-axis of component A nearly perpendicular to the field. In turn, by tilting $\mathrm{RD}$ at $-19^{\circ}$, the specimen is mounted in a position where the $c$-axis of component $\mathrm{B}$ is perpendicular to the magnetic field. The magnetic annealing of samples in both tilt-positions results in the increase of the texture peak corresponding to grains with the $c$-axis perpendicular to the field, while the other texture component disappears completely. The observed change in the type of texture can be understood as a result of selective grain growth induced by additional driving force created by the anisotropy of the magnetic susceptibility of $\mathrm{Zn}$. The origin of this driving force was considered by Mullins [1]. If the volume density of the magnetic free energy $\omega$ in a crystal induced by a uniform magnetic field is independent on crystal shape and size (the condition for this is $\chi \ll 1)$ then the magnetic driving force acting on the boundary of two crystals that have different magnetic susceptibilities is given by:

$p=\omega_{1}-\omega_{2}=\frac{\mu_{0} H^{2}}{2}\left(\chi_{1}-\chi_{2}\right)$,

where $\chi_{1}$ and $\chi_{2}$ are the susceptibilities of crystal 1 and 2 , respectively, along the magnet field $H$. For the case of zinc, Eq. (1) is transformed to

$p=\mu_{0} \frac{\Delta \chi}{2} H^{2}\left(\cos ^{2} \theta_{1}-\cos ^{2} \theta_{2}\right)$,

where $\theta_{1}$ and $\theta_{2}$ are the angles between the direction of magnetic field and the hexagonal (or $c$ or $\langle 0001\rangle$ axis) in both neighboring grains, $\Delta \chi$ is the difference in susceptibilities parallel and perpendicular to the hexagonal axis. The force $p$ is directed towards the grain with smaller value of $\theta$ and does not depend on the sign of the magnetic field. The magnitude of the difference in the magnetic free energy of different grains in investigated 
$\mathrm{Zn}-1.1 \% \mathrm{Al}$ alloy can be estimated using the measurements of the crystal diamagnetism of $\mathrm{Zn}$ crystals [3]. According to [3] the gram susceptibility of $\mathrm{Zn}$ parallel and perpendicular to hexagonal (or $c$ or $\langle 0001\rangle$ ) axes are $\chi_{\|}=-0.190 \times 10^{-6} \mathrm{~cm}^{3} /$ $\mathrm{g}$ and $\chi_{\perp}=-0.145 \times 10^{-6} \mathrm{~cm}^{3} / \mathrm{g}$, respectively. Conversion of these data from Gauss unit system gives for volume susceptibility in SI units as $\chi_{\|}=$ $-1.695 \times 10^{-5}$ and $\chi_{\perp}=-1.294 \times 10^{-5}$. According to Eq. (1) the maximum magnetic driving force grain growth in $\mathrm{Zn}$ in the case of the field strength of $2.55 \times 10^{7} \mathrm{~A} / \mathrm{m}$ and the difference in volume susceptibilities of $\Delta \chi=\chi_{\perp}-\chi_{\|}=0.401 \times 10^{-5}$ is $p_{\max }=1.65 \mathrm{~kJ} / \mathrm{m}^{3}$. This force is related to a bicrystal with the angle of $90^{\circ}$ between basal planes (or $\langle 0001\rangle$ directions) oriented parallel and perpendicular to the field direction. For boundaries with other disorientation the driving force should be lower. It is reasonable to expect that grains corresponding to texture components $\mathrm{A}$ and $\mathrm{B}$ should have the highest proportion of common boundaries with disorientation angles ranging between $30^{\circ}$ and $40^{\circ}$ and TD as the rotation axis (Figs. 1 and 2). If the $c$-axis of the grains of one texture component is oriented perpendicular to the field, then these grains experience an additional driving force for the growth (or for the motion of their boundaries) in the direction of grains of another component. In this case the orientation of the $c$-axis of the second component, with respect to the field direction, ranges between $50^{\circ}$ and $60^{\circ}$ and the magnetic force according to Eq. (1) varies from 0.4 to $0.7 \mathrm{~kJ} / \mathrm{m}^{3}$. This force can be compared with usual capillary driving force for grain growth determined as

$p_{\mathrm{c}}=\frac{2 \sigma}{R}$,

where $\sigma$ is grain boundary energy, $R$ is mean grain radius. The value of the ratio of the average grain boundary curvature to the inverse of the mean linear grain intercept has been experimentally found for $\mathrm{Al}$ as 0.31 throughout grain growth [4]. Applying the same relationship for $\mathrm{Zn}$ alloy with mean grain size of $150 \mu \mathrm{m}$ we obtain $R \cong 0.5 \mathrm{~mm}$. Assuming a grain boundary energy of typically $0.3 \mathrm{~J} / \mathrm{m}^{2}$ [5], the capillary force amounts to $p_{\mathrm{c}} \cong 1.2$ $\mathrm{kJ} / \mathrm{m}^{3}$. Thus, a comparison of the respective driving forces reveals that the magnetic force is at least of the order of the capillary forces and able to make a strong influence on grain growth increasing the growth rate of those grains whose $\langle 0001\rangle$ axis is perpendicular to the field. When the sample in our experiment is tilted at $+19^{\circ}$ the $c$-axis of component $\mathrm{A}$ becomes almost perpendicular to the field and the $c$-axis of component $\mathrm{B}$ is at $51-56^{\circ}$ in respect to the field. Magnetic free energy of grains A reaches its minimum and becomes lower than the energy of all other orientations. Consequently, the additional magnetic driving force arises and enhances the growth of grains A. At the same time texture component $\mathrm{B}$ disappears. The growth in intensity of grains $\mathrm{A}$ is close to the drop in intensity of grains B. Therefore, it is reasonable to suggest that the disappearance of grains B is related to the growth of grains $\mathrm{A}$, which grow mainly at the expense of grains B. When the sample is tilted at $-19^{\circ}$ to the field, the $c$-axis of component $\mathrm{B}$ becomes perpendicular to the field and grains B grow at the expense of grains A. In addition, some growth can naturally occur at the expense of other orientations. In a position where the sample is parallel to the field direction, the $c$ axes of both components are tilted at $70-75^{\circ}$ to the field. The magnetic free energy of the grains of both components is equal and neither grains A nor grains $\mathrm{B}$ have additional force to grow. The experiment reveals that although the intensity of texture subcomponents after magnetic annealing in the orientation of RD parallel to the field (Fig. 1(a)) remains almost the same as the intensity of texture components before the annealing, their $c$ axes become closer to the normal direction. Such behavior can be explained by preferable growth of those grains within the same component whose $c$ axis is closer to the normal direction of the field that results in some redistribution in texture intensity to the normal direction of the sheet.

\section{Concluding remarks}

For the first time, it has been demonstrated that annealing of zinc alloy sheet in a high magnetic field can make significant changes in crystallographic texture. Depending on the orientation of specimen to the applied field the texture components can be 
strengthened, disappear or retain the original intensity. The intensity of the components related to the grain orientations with lowest magnetic susceptibility increases during magnetic annealing whereas components related to higher susceptibility completely disappear. The obtained results are interpreted in terms of selective grain growth exerted by magnetic driving force for boundary migration.

\section{Acknowledgements}

This work has been supported by the US Army Grant DAAD 19-99-1-0311. D.A. Molodov con- veys his thanks to the Deutsche Forschungsgemeinschaft for financial support. Critical reading of the manuscript by Prof. Günter Gottstein is gratefully acknowledged.

\section{References}

[1] Mullins WW. Acta Metall 1956;4:421.

[2] Molodov DA, Gottstein G, Heringhaus F, Shvindlerman LS. Acta Mater 1998;46:5627.

[3] Mac Clure JW, Marcus JA. Phys Rev 1951;84:787.

[4] Patterson BR, Liu Y. Metall Trans A 1992;23:2481.

[5] Antonov AV, Shvindlerman LS. Sov Phys Solid State 1973; $15: 1083$. 